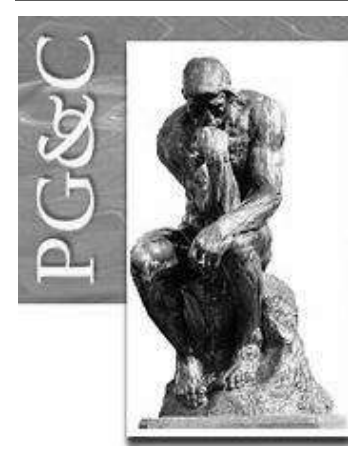

\title{
GESTÃO DO CONHECIMENTO E A MINERAÇÃO: PROPOSTA DE FRAMEWORK COMO ESTRATÉGIA DE NIVELAMENTO DOS CONHECIMENTOS ENTRE COLABORADORES DO MESMO CARGO
}

\author{
Amanda Braga de Almeida \\ Graduanda em Engenharia de Produção pela Universidade Federal de \\ Ouro Preto, Brasil. \\ E-mail: amanda.braga@aluno.ufop.edu.br \\ Alana Deusilan Sester Pereira \\ Doutora em Administração de Empresas pela Fundação Getúlio Vargas, \\ Brasil. Professora da Universidade Federal de Ouro Preto, Brasil. \\ E-mail: alana@ufop.edu.br \\ Renan Araújo Macieira \\ Graduado em Engenharia Elétrica pelo Centro Universitário de Minas \\ Gerais, Brasil. \\ E-mail: renan.macieira@gmail.com
}

\begin{abstract}
Resumo
O estudo objetiva propor um framework teórico-analítico que suscite reflexões de como as mineradoras podem implantar a gestão do conhecimento para auxiliar no nivelamento de conhecimentos dos colaboradores do setor de Manutenção em Instrumentação e Automação. Especificamente, pretendese: (i) mapear na literatura trabalhos sobre a gestão do conhecimento na mineração; (ii) identificar como o Modelo de Referência da Sociedade Brasileira de Gestão do Conhecimento pode atender o caso; e (iii) elaborar um esquema teórico-analítico apresentando as etapas para implantação da gestão do conhecimento neste setor. Para tanto, realizaram-se uma pesquisa bibliográfica e inspiração em estudo de caso. Na coleta de dados, empregou-se as entrevistas com gestores; análise em documentos institucionais e observação in loco. Assim, constatou-se que os trabalhos não abordam as etapas de implantação da gestão do conhecimento em empresas mineradoras. Ademais, não se aprofundam na discussão de quais conhecimentos deveriam ser gerenciados. Nesse sentido, visando reduzir essa lacuna, pressupõe-se que as organizações devem reconhecer quais conhecimentos devem ser trabalhados e quais sujeitos os necessitam. Tem-se, portanto, que diagnosticar os desafios e problemas de gestão do conhecimento, mapear os processos críticos, definir cada atividade e cargos envolvidos, identificar o processo de gestão do conhecimento a ser priorizado e selecionar e implementar práticas de gestão do conhecimento; sendo etapas cruciais para as organizações alcançarem seus objetivos de conhecimento e melhores resultados. Sugere-se para estudos futuros o acompanhamento e avaliação destas práticas de gestão do conhecimento.
\end{abstract}

Palavras-chave: Conhecimento Organizacional. Gestão do Conhecimento. Mineração.

\section{KNOWLEDGE MANAGEMENT IN MINING: A STRATEGIC FRAMEWORK FOR LEVELING KNOWLEDGE AMONG EMPLOYEES WITH THE SAME POSITION}

\begin{abstract}
The study proposes a theoretical-analytical framework that inspires reflection on how mining companies can implement knowledge management to aid in leveling up the knowledge of employees in the instrumentation and automation maintenance sector. Specifically, we seek to (i) map the works in the
\end{abstract}

Perspectivas em Gestão \& Conhecimento, João Pessoa, v. 11, número especial, p. 97-114, mar. 2021. DOI: http://dx.doi.org/10.22478/ufpb.2236-417X.2021v11nEspecial.57314

http://periodicos.ufpb.br/ojs2/index.php/pgc. ISSN: 2236-417X. Publicação sob Licença (cc) EY-Nc-ND 
literature on knowledge management in mining; (ii) identify how the Brazilian Knowledge Management Society's Reference Model addresses the case; and (iii) devise a theoretical-analytical scheme outlining the steps for implementing knowledge management in this sector. To this end, we carried out a bibliographic search and partial case study. Data collection comprised of interviews with managers, analysis of institutional documents, and observation in loco. We found that the works do not address the stages of implementing knowledge management in mining companies. Furthermore, they do not broaden the discussion of precisely what knowledge should be managed. Thus, in order to reduce this gap, we assume that organizations should recognize what knowledge should be developed and which subjects need it. Therefore, we seek to assess the challenges and problems of knowledge management, map the critical processes, define each activity and the position involved, identify the knowledge management process to be prioritized, and select and implement knowledge management practices. Indeed, these are crucial steps for organizations to achieve their knowledge goals and better results. For future studies, we suggest the ongoing monitoring and evaluation of these knowledge management practices.

Keywords: Organizational Knowledge. Knowledge Management. Mining.

\section{INTRODUÇÃO}

Sendo o setor da mineração um dos principais pilares econômicos nacionais, o investimento em práticas de Gestão do Conhecimento (GC) nas mineradoras deveria ser intenso, visto que tornam possível otimizar os processos e a fabricação de produtos ou serviços nas organizações. Ainda, para Teixeira Filho (2000), o conhecimento torna-se um motor econômico em um mercado de alto dinamismo e competitividade.

Nesta perspectiva, Nonaka e Takeuchi (1997) ressaltam que o sucesso das organizações está intimamente relacionado à sua capacidade de criar novos conhecimentos e os disseminar. Atrelado a esse pensamento, segundo Boikanyo et al. (2016), inúmeras organizações têm dificuldade em identificar o conhecimento que é relevante para o seu crescimento e nem todas utilizam suas estratégias orientadas à GC, além de deixarem a desejar quanto à formalização e nivelação do conhecimento proveniente das interações dos funcionários (BRAGA et al., 2014).

Isso ocorre pois, conquanto a colaboração se faça presente na consulta à equipe na tomada de decisões, a falta da documentação dessa troca de conhecimentos informal pode acarretar no conflito de informações, conforme observado por Mishra et al. (2018). Ademais, embora em parte das empresas sejam disponibilizados materiais para consulta, como manuais e procedimentos, a experiência ainda é a maior fonte de conhecimento nas indústrias de mineração (PEREIRA; BARBOSA, 2018).

Nesse cenário, ressalta-se a indispensabilidade do uso de estratégias de nivelamento do conhecimento para que seja possível reter, nivelar e disseminar o conhecimento rumo a vantagem competitiva, pois, para Nonaka e Takeuchi (2003), o conhecimento é o único capaz de trazer esse diferencial, dada sua superioridade diante de ativos físicos e financeiros. Para Ceribeli e Aguiar (2015) e Makhubela e Ngoepe (2018), entre os benefícios de se nivelar o conhecimento, evidenciam-se a promoção da melhoria contínua do uso de recursos, através da prevenção e diminuição do tempo de resposta aos problemas, bem como a redução de custos e perdas de informações com a rotatividade de funcionários. Além disso, faz-se possível a utilização do conhecimento para aumentar a produtividade dos colaboradores, por meio da capacitação e minimização de retrabalhos.

Além dos meios tradicionais mostrados por Braga et al. (2014) e Makhubela e Ngoepe (2018), como reuniões registradas em atas e entrevistas nas saídas dos funcionários, entre as práticas de GC utilizadas para nivelar o conhecimento, destacam-se os modelos interativos de

Perspectivas em Gestão \& Conhecimento, João Pessoa, v. 11, número especial, p. 97-114, mar. 2021. 
lições aprendidas contendo causas e soluções dos problemas (BRAZÃO; PECH, 2018) e as bases de dados, que permitem a geração de relatórios de desempenho, rastreio dos processos e a comunicação formal (CERIBELI; AGUIAR, 2015). Ademais, há iniciativas de implementação de mecanismos que capturam o conhecimento tácito através de padrões de comportamento e o transformam em conhecimento explícito (DELGADO et al., 2014), bem como ferramentas que sugerem equipes produtivas com boa relação interpessoal, atrelando quem necessita do aprimoramento à quem possui as habilidades (FONSECA et al., 2013).

Logo, para estabelecer o nivelamento eficiente nas indústrias mineradoras, faz-se necessário promover uma cultura do compartilhamento do conhecimento, visto que alguns colaboradores não se sentem confortáveis para transferir seu conhecimento adquirido em anos de experiência. Não obstante, o medo de serem dispensados logo após a transferência de conhecimentos foi destacado como um dos motivos do não compartilhamento, conforme Pereira e Barbosa (2018) que, em seu trabalho, utilizaram uma amostra composta por trabalhadores da mesma organização estudada nesta pesquisa.

Kukko (2013) acrescenta que o compartilhamento do conhecimento contribui para o desempenho institucional, dado seus principais desafios: profissionais com pouco tempo de atuação e perda dos que possuem experiência e conhecimento técnico. Assim, torna-se evidente que a implementação de estratégias de nivelamento deve ser realizada com a integração dos colaboradores e o estímulo ao engajamento em todo o processo.

Diante do exposto, é possível constatar que os trabalhos não abordam as etapas de implantação da GC em empresas mineradoras. Com a finalidade de reduzir essa lacuna, este trabalho busca responder: como a gestão do conhecimento pode ser implantada para auxiliar no nivelamento dos conhecimentos entre colaboradores do mesmo cargo na Supervisão de Manutenção em Instrumentação em uma empresa do setor de mineração?

Com isso, o principal objetivo é, a partir da literatura pertinente, propor um framework teórico-analítico que possa suscitar reflexões de como as organizações podem implantar uma estratégia de GC que auxilie no nivelamento de conhecimentos dos colaboradores da área de instrumentação em uma organização mineradora. Para tanto, especificamente, pretende-se: (i) mapear na literatura trabalhos sobre a GC no setor de mineração; (ii) identificar como o modelo MR-SBGC pode ser adaptado ao contexto do caso selecionado; e (iii) elaborar um esquema teórico-analítico que apresente as etapas para implantação da GC no setor estudado.

A relevância desta pesquisa ancora-se na dificuldade de se encontrar estudos de GC na mineração, observada até mesmo por autores como Boikanyo et al. (2016), que ressaltam tal escassez como um agravante na avaliação do nível de GC no setor da mineração. Decorrente deste problema, o presente estudo mostra-se importante por contribuir nos âmbitos teórico e prático. Logo, as contribuições teóricas se dão por propiciar o diálogo entre autores de GC, em perspectiva processual, quanto ao nivelamento dos conhecimentos entre colaboradores em uma empresa do setor de mineração. Ademais, será possivel acrescentar à teoria uma estrutura de identificação e operacionalização de conhecimentos críticos, em conjunto com a proposta de uma estratégia para nivelar e compartilhar conhecimentos, que possibilite avanços nas recomendações de estudos futuros e na literatura de GC.

Somam-se a isto os seguintes efeitos contributivos em âmbito empresarial, como exemplo: por meio da descrição dos conhecimentos críticos dos cargos na área de instrumentação, cada colaborador saberá quais habilidades precisa obter caso almeje futuras promoções. Além disso, em decorrência da percepção da necessidade de se investir na criação, sistematização e compartilhamento do conhecimento, para que todos empregados de um mesmo cargo estejam nivelados e saibam realizar as atividades da mesma forma, haverá sempre continuidade no processo. Ademais, será propiciado o auxílio à disseminação dos conhecimentos adquiridos em treinamentos, eventos corporativos e experiências vividas; bem como à análise e gerenciamento dos riscos, incluindo a agilidade na solução de problemas

Perspectivas em Gestão \& Conhecimento, João Pessoa, v. 11, número especial, p. 97-114, mar. 2021. 
relacionados aos diagnósticos de equipamentos e às oscilações anormais dos processos. Com isso, a pesquisa suscitará reflexões empíricas pelos colaboradores e gestores sobre a relevância da $\mathrm{GC}$, no que tange às melhorias dos procedimentos da área e às tomadas de decisão, de forma a incentivar o engajamento no trabalho em equipe, acarretando no alinhamento aos interesses da empresa, uma vez que todos os profissionais passam a colaborar entre si e com toda a organização.

\section{REFERENCIAL TEÓRICO}

\subsection{Gestão do Conhecimento}

Segundo Giddens (1991), a percepção da contribuição trazida pela GC evidencia-se a partir dos anos 90, impulsionada pelo deslocamento da sociedade de bens/produtos para a sociedade do conhecimento. Nesse seguimento, tem-se tornado cada vez mais claro que a cultura das organizações e as pessoas são cruciais para determinar o sucesso das iniciativas de gestão do conhecimento, mostrando que a eficiência da gestão vai além das tecnologias para compartilhamento do conhecimento (RUBENSTEIN-MONTANO et al., 2001).

Nonaka e Takeushi (1997) afirmam que o conhecimento pode ser caracterizado como tácito ou explícito. Os tácitos são os conhecimentos subjetivos, individualizados e divididos em duas dimensões: técnica (saber fazer) e cognitiva (crenças, valores, modelos mentais, entre outros). Tal conhecimento está associado à capacidade de agir das pessoas, às suas habilidades, expertises, experiências, competências, raciocínio, conclusões, geração de ideias, opiniões, emoções, intuição e criatividade. Já o conhecimento explícito pode ser entendido como a materialização ou a representação do conhecimento de alguém a partir de algum tipo de registro, escrita ou mídia, que possa ser transferido, transmitido, comunicado, visualizado e armazenado (NONAKA; TAKEUSHI, 1997).

Adiante, para Probst, Raub e Romhardt (2002), o conhecimento intangível precisa ser zelosamente estruturado em seu processo de seleção e armazenagem, além de ser atualizado para gerar valor. Logo, considerando a necessidade de se gerenciar tal ativo, o termo gestão do conhecimento (GC) passa a ser mais bem discutido. Para Chait (1999), a GC é o processo ideal para maximizar e disponibilizar os conhecimentos existentes na organização e fazer com que todo conhecimento valioso esteja à disposição das pessoas que constituem a empresa. Assim, o foco da GC é: (a) aprendizado individual e organizacional (cultura organizacional); (b) desenvolvimento de competências individuais e organizacionais; (c) conectividade entre as pessoas; e (d) mapeamento, codificação e compartilhamento do conhecimento organizacional (TERRA, 2005, p. 81).

Portanto, de acordo com Teixeira Filho (2000, p. 22), "a gestão do conhecimento pode ser vista como uma coleção de processos que governa a criação, disseminação e utilização do conhecimento para atingir plenamente os objetivos da organização".

E, em consonância, para Terra $(2005$, p. 8):

[...] gestão do conhecimento significa organizar as principais políticas, processos e ferramentas gerenciais e tecnológicas a luz de uma melhor compreensão dos processos de geração, identificação, validação, disseminação, compartilhamento, proteção e uso dos conhecimentos estratégicos para gerar resultados (econômicos) para a empresa e benefícios para os colaboradores internos e externos (stakeholders).

\subsubsection{Nivelamento de Conhecimentos}

Também encontrado na literatura como difusão interativa do conhecimento, o termo nivelamento do conhecimento refere-se à disseminação do conhecimento organizacional criado para indivíduos da mesma divisão, divisões distintas ou externos à entidade (NONAKA e TAKEUCHI, 1997). Portanto, Takeuchi e Nonaka (2008) consideram o nivelamento transversal do conhecimento como o quinto e último subprocesso de criação do conhecimento 
organizacional. Dessa forma, torna-se um processo de atualização contínua, dadas as constantes mudanças que envolvem a criação do conhecimento.

Ainda, Takeuchi e Nonaka (2008) afirmam que o contexto promotor é o que garante a criação eficaz do conhecimento. Nesse sentido, consideram dois promotores intimamente ligados ao incentivo do nivelamento do conhecimento, sendo estes a introdução de uma visão do conhecimento e a globalização do conhecimento local. Entretanto, para ambos autores, todos os cinco promotores influenciam no nivelamento, visto que diminuem os desafios da comunicação e elevam a disseminação da informação.

Para Carvalho (2012), o nivelamento do conhecimento objetiva compartilhar e reafirmar o conhecimento criado, o que torna desejável que as conversas relacionadas sejam de confirmação do conhecimento. Logo, percebe-se que: em conjunto com a transferência do conhecimento, o nivelamento cria eficiência no interior da organização. Todavia, para que ocorra sua implementação, é imprescindível que sejam fomentados valores comuns, resultados mutuamente adequados e o encorajamento aos interesses partilhados (HITT et al., 2001A; QUINN et al., 1996). Não obstante, Stefanovitz e Nagano (2006) ressaltam a importância da documentação voltada ao aprendizado para o nivelamento do conhecimento, quanto à diminuição da concentração excessiva dos novos conhecimentos apenas em seus criadores e à sua incorporação efetiva na organização.

\subsubsection{Modelos de Gestão do Conhecimento}

Modelos de GC são um guia para a tomada de decisões organizacionais, abordando os principais conceitos, princípios e elementos de uma área do conhecimento, em uma visão holística (WEBER et al., 2002). Tais modelos podem ser classificados em três dimensões: prescritivos, descritivos ou híbridos. Para Rubenstein-Montano et al. (2001), os modelos prescritivos orientam os procedimentos através da sugestão de uma metodologia de GC a ser seguida. Ainda, os descritivos constatam particularidades que influenciam de forma positiva ou negativa no sucesso da implementação da GC. Já os híbridos, para Weber et al. (2002), sugerem como as coisas devem ser feitas de maneira prática, com a junção dos dois modelos.

A literatura conta com mais de 160 modelos de GC, também chamados de estrutura ou framework. Abaixo, o Quadro 1 explicita alguns desses modelos:

Quadro 1 - Modelos de Gestão do Conhecimento

\begin{tabular}{|c|c|}
\hline Autores & \multicolumn{1}{|c|}{ Modelos de Gestão do Conhecimento } \\
\hline Probst, Raub e & $\begin{array}{r}\text { Há 8 elementos construtivos da GC: seis essenciais (identificação, aquisição, } \\
\text { desenvolvimento, partilha e distribuição, utilização, preservação) e dois } \\
\text { estratégicos (metas e avaliação). A ordem a seguir inicia-se nas metas do } \\
\text { conhecimento, nas seis etapas essenciais e, por fim, na avaliação. }\end{array}$ \\
\hline Spenhardt (2002) & $\begin{array}{r}\text { O Spender Levels distingue o conhecimento individual e coletivo e combina-o } \\
\text { com a classificação (tácito ou explícito), para descobrir onde o mesmo reside. Há } \\
4 \text { vertentes: conhecimento consciente, objetivado, automático e coletivo. }\end{array}$ \\
\hline Angeloni (2008) & $\begin{array}{c}\text { 3 dimensões interdependentes se interagem: infraestrutura organizacional } \\
\text { (visão holística, estrutura, cultura e estilo de gerência), pessoas (intuição, } \\
\text { compartilhamento, aprendizagem, criatividade, inovação e modelos mentais) e } \\
\text { tecnologia (workflow, gerenciamento eletrônico de documentos, redes, data }\end{array}$ \\
\hline
\end{tabular}

Perspectivas em Gestão \& Conhecimento, João Pessoa, v. 11, número especial, p. 97-114, mar. 2021. 


\begin{tabular}{|c|c|}
\hline Stewart (1998) & $\begin{array}{l}\text { Medidas de capital humano agregam valor para o cliente e relacionam-se aos } \\
\text { colaboradores especializados e suas atividades. Resultados mensurados pela: } \\
\text { inovação, aprendizado individual, experiência, rotatividade, posição hierárquica } \\
\text { e atitudes dos funcionários. O capital intelectual é formado por: humano, } \\
\text { estrutural e de clientes; com seus respectivos conhecimentos e competências. }\end{array}$ \\
\hline $\begin{array}{l}\text { Davenport e Pruzak } \\
\qquad(2003)\end{array}$ & $\begin{array}{l}\text { Deve-se possuir infraestrutura técnica e organizacional, orientação por } \\
\text { processos, canais para disseminação do conhecimento, apoio da alta } \\
\text { administração e motivações não triviais. A cultura deve ser voltada para o } \\
\text { conhecimento de fácil compreensão. }\end{array}$ \\
\hline Teixeira Filho (2000) & $\begin{array}{l}\text { Possui as dimensões do conhecimento (tácito e explícito), tecnologia (apoio e } \\
\text { integração) e organização (cultura e estratégia). Há a preparação, explicitação, } \\
\text { socialização, divulgação e avaliação. O conhecimento é avaliado pelas ações } \\
\text { subsequentes, advém de informações e os criadores devem ser qualificados. }\end{array}$ \\
\hline $\begin{array}{l}\text { Bukowitz e Williams } \\
\qquad(2002)\end{array}$ & $\begin{array}{l}\text { Sugere uma tipologia com ênfase na capacidade de transmitir o conhecimento, } \\
\text { a partir de um diagnóstico de GC que conta com } 7 \text { seções de reestruturação: } \\
\text { obtenha, utilize, aprenda, contribua, avalie, construa/mantenha, despoje. }\end{array}$ \\
\hline Wiig (1993) & $\begin{array}{l}\text { O conhecimento é organizado em redes semânticas para poder ser utilizado. } \\
\text { Tais redes relacionam conceitos em estudo. Possui } 4 \text { vertentes: criação e } \\
\text { alimentação, compilação e transformação, disseminação e aplicação, e a } \\
\text { realização de valor. }\end{array}$ \\
\hline Kaplan e Norton (1997) & $\begin{array}{l}\text { Balanced Scorecard (BSC) é um método de avaliação que propõe o uso de } \\
\text { indicadores e vetores de desempenho dos processos de GC. Possui foco no } \\
\text { conhecimento que pode ser abordado no planejamento estratégico. }\end{array}$ \\
\hline $\begin{array}{c}\text { Nonaka e Takeuchi } \\
\text { (1997) }\end{array}$ & $\begin{array}{l}\text { A conversão do conhecimento tácito para explícito ocorre com a: socialização, } \\
\text { externalização, combinação e internalização (SECI). Há } 5 \text { fases para criar o } \\
\text { conhecimento: compartilhamento do conhecimento tácito, criação de } \\
\text { conceitos, sua justificação, construção de um arquétipo e a disseminação } \\
\text { interativa do conhecimento. Os profissionais devem ser qualificados, possuir } \\
\text { experiências e habilidade de comunicação, promovendo o trabalho em equipe e } \\
\text { debates. }\end{array}$ \\
\hline Edvisson (1998) & $\begin{array}{l}3 \text { componentes: capital humano (capacidade individual de atuação a partir das } \\
\text { habilidades, experiências, valores e educação formal), capital organizacional } \\
\text { (patentes, conceitos, modelos administrativos e informatizados) e capital de } \\
\text { clientes (parceiros, fornecedores e a imagem que a organização passa). }\end{array}$ \\
\hline
\end{tabular}




\begin{tabular}{|c|c|} 
Sicsú e Dias (2005) & $\begin{array}{r}\text { Possui os princípios: cultura de aprendizagem, planejamento estratégico, } \\
\text { colaboração, manutenção de recursos humanos qualificados, condições } \\
\text { tecnológicas adequadas, foco nos sistemas de informações, futuro condicionado } \\
\text { pela história da empresa, inovação e modernização. }\end{array}$ \\
\hline Terra (2005) & $\begin{array}{r}\text { Sete dimensões do conhecimento: fatores estratégicos e alta administração, } \\
\text { cultura organizacional, estrutura organizacional, políticas e práticas da gestão de } \\
\text { recursos humanos, sistemas de informações, mensuração de resultados e } \\
\text { aprendizado com o ambiente. Há componentes relacionados à estrutura, } \\
\text { indivíduos e ambiente externo. }\end{array}$ \\
\hline
\end{tabular}

\subsubsection{Modelo de Referência da SBGC}

Saito e Fukunaga (2020) relatam que, a partir do intuito de originar um modelo que contribuísse com as organizações e pudesse ser aplicado em diversos casos, em 2013, foi criada de forma colaborativa a primeira versão do Modelo de Referência SBGC (MR-SBGC). Ainda, ao longo dos anos, o modelo ganhou várias atualizações, de forma a proporcionar uma visão integrada de alto nível da gestão quanto ao conhecimento organizacional.

Para tanto, basearam-se em práticas de mercado e na teoria. Assim, o modelo possibilita a avaliação do grau de maturidade de um sistema de GC e o direcionamento quanto às práticas e iniciativas que podem ser implementadas (SAITO; FUKUNAGA, 2020).

\begin{tabular}{|c|c|c|c|c|}
\multicolumn{1}{c|}{} & \multicolumn{2}{c|}{ Quadro 2 - Modelo de Referência SBGC Expandido } \\
\cline { 2 - 5 } & Dimensões & \multicolumn{3}{c|}{ Variáveis } \\
\cline { 2 - 5 } $\begin{array}{c}\text { GC deve apoiar o } \\
\text { negócio }\end{array}$ & $\begin{array}{c}\text { Alinhamento } \\
\text { com o Negócio }\end{array}$ & $\begin{array}{c}\text { Estratégia de } \\
\text { GC }\end{array}$ & $\begin{array}{c}\text { Conhecimentos } \\
\text { Relevantes }\end{array}$ & $\begin{array}{c}\text { Governança } \\
\text { da GC }\end{array}$ \\
\cline { 2 - 5 } $\begin{array}{c}\text { GC exige } \\
\text { ambiente } \\
\text { facilitador }\end{array}$ & $\begin{array}{c}\text { Iniciativas e } \\
\text { Práticas de GC }\end{array}$ & $\begin{array}{c}\text { Processos de } \\
\text { Conhecimento }\end{array}$ & $\begin{array}{c}\text { Abordagens em } \\
\text { GC }\end{array}$ & $\begin{array}{c}\text { Práticas de } \\
\text { GC }\end{array}$ \\
\cline { 2 - 5 } & Facilitador & Organizacional & Elementos de \\
Gestão & Infraestrutura \\
\hline
\end{tabular}

Fonte: Adaptado de Saito e Fukunaga (2020)

Logo, o modelo foi dividido em três dimensões (Quadro 2): Alinhamento com o Negócio; Iniciativas e Práticas de GC e Ambiente Facilitador. Além disso, há duas premissas a ser seguidas, sendo a exigência de um ambiente facilitador para a GC e a necessidade do amparo da mesma ao negócio. Isso ocorre pois, há diversos conhecimentos organizacionais que devem ser gerenciados, mas poucos recursos para isso, tornando necessária a priorização. Além disso, para facilitar a GC, a cultura organizacional deve ser orientada para a colaboração e a criação do conhecimento (SAITO; FUKUNAGA, 2020).

Além do mais, Saito e Fukunaga (2020) mostram que a dimensão do Alinhamento com o Negócio possui três variáveis: (i) Estratégia de GC, que agrupa atividades que priorizam os objetivos de conhecimento, por meio da identificação dos objetivos estratégicos e dos desafios 
enfrentados pelo negócio, bem como os problemas de conhecimento que influenciam em tais desafios; (ii) Conhecimentos Relevantes, que são identificados através do mapeamento de conhecimento crítico, dados os critérios de risco e relevância, de forma a priorizá-los para resolver os problemas de conhecimento; e (iii) Governança da GC, onde são definidos os meios para acompanhar os resultados da GC, quais os patrocinadores, stakeholders e responsáveis por sua implementação e gestão nos níveis tático e operacional.

No que tange a dimensão das Iniciativas e Práticas de GC, os autores apresentam os: (i) Processos de Conhecimento, relacionados à criação, transferência, retenção e aplicação do conhecimento, cuja escolha do que se realizar depende dos objetivos de GC; (ii) Abordagens em GC, que auxiliam no alinhamento da criação de iniciativas de GC e na avaliação da contribuição das práticas já utilizadas, além de apoiar a comunicação em vários níveis organizacionais; e (iii) Práticas de GC, que visam solucionar os problemas de conhecimento.

Já quanto a dimensão do Ambiente Facilitador, eles apontam a (i) Cultura Organizacional, que deve ser orientada para a colaboração no processo de criação do conhecimento, de forma a estar alinhada com os desempenhos das iniciativas de GC; (ii) Elementos da Gestão, que aborda como são feitas as escolhas relacionadas à tomada de decisão, motivação dos funcionários e parceiros, e a definição de objetivos no âmbito da coordenação; e (iii) Infraestrutura, que trata da estrutura física e tecnológica para proporcionar a interação entre os colaboradores e o suporte às práticas de GC, respectivamente.

\subsubsection{Práticas de Gestão do Conhecimento}

Inúmeras são as práticas de GC que propiciam estrategicamente a produção, retenção, disseminação, compartilhamento e aplicação do conhecimento, seja no recinto das organizações, seja na relação das mesmas com o mundo ao seu redor (BATISTA et al., 2005). Tais práticas devem ser escolhidas para utilização de acordo com o objetivo que se pretende alcançar, podendo contar com o apoio de tecnologias de GC.

Nesse âmbito, Carvalho (2012) alega que o maior desafio das organizações é identificar as práticas do conhecimento já presentes em seu ambiente, além de atrelá-las a novas práticas para atingir o propósito organizacional. Ainda, Batista et al. (2005) classificam as práticas em três agrupamentos: as relacionadas aos aspectos de gestão de recursos humanos, as ligadas à estruturação dos processos organizacionais e as cujo foco central é a base tecnológica e funcional. Todavia, os autores têm consciência de que as classificações estabelecidas são arbitrárias, pois várias práticas podem transcendê-las.

Diante do exposto, o Quadro 3 elucida os conceitos relacionados à algumas práticas de GC presentes entre as mais de 32 citadas na literatura:

Quadro 3 - Práticas de Gestão do Conhecimento

\begin{tabular}{|c|c|}
\hline Práticas & Conceito \\
\hline Gestão de Conteúdo & $\begin{array}{c}\text { Envolve pesquisa contínua de conteúdos, como meio de selecionar, } \\
\text { capturar, classificar, indexar, registrar e depurar informações. }\end{array}$ \\
\hline (BATISTA et al., 2005) & $\begin{array}{c}\text { Criação de estruturas coletivas de referência, mudanças na base de } \\
\text { conhecimento e aumento da capacidade de resolver problemas. }\end{array}$ \\
\hline
\end{tabular}

Perspectivas em Gestão \& Conhecimento, João Pessoa, v. 11, número especial, p. 97-114, mar. 2021. 


\begin{tabular}{|c|c|}
\hline $\begin{array}{l}\text { Benchmarking } \\
\text { (SPENDOLINI, 1994) }\end{array}$ & $\begin{array}{l}\text { Consiste em medir os processos, produtos e serviços de uma } \\
\text { organização e, compará-los com os de outras empresas. }\end{array}$ \\
\hline $\begin{array}{l}\text { Coaching } \\
\text { (OLIVEIRA, 2018) }\end{array}$ & $\begin{array}{c}\text { Aconselhamento e consultoria liderada pelo treinador, visando o } \\
\text { desenvolvimento pessoal e profissional. }\end{array}$ \\
\hline $\begin{array}{l}\text { Comunicação Institucional } \\
\qquad \text { (REGO, 1986) }\end{array}$ & $\begin{array}{c}\text { Reunião das partes distintas da empresa, produzindo as condições } \\
\text { para um trabalho coordenado de estruturas e atingindo as metas } \\
\text { programadas. }\end{array}$ \\
\hline $\begin{array}{l}\text { Comunidades de Prática } \\
\text { (TERRA, 2005) }\end{array}$ & $\begin{array}{c}\text { Complemento às estruturas formais que prosperam em organizações } \\
\text { onde há estágio elevado de confiança entre os gestores e os } \\
\text { colaboradores. }\end{array}$ \\
\hline $\begin{array}{l}\text { Educação Corporativa } \\
\text { (BATISTA et al., 2005) }\end{array}$ & $\begin{array}{l}\text { Atualização uniforme em todas as áreas a partir da educação } \\
\text { continuada, por meio de sistemas de ensino a distância, universidade } \\
\text { corporativa, etc. }\end{array}$ \\
\hline $\begin{array}{l}\text { Gestão de Competências } \\
\text { (BRANDÃO e GUIMARÃES, 2001) }\end{array}$ & $\begin{array}{c}\text { Modo de planejar, organizar, desenvolver, acompanhar e avaliar as } \\
\text { competências organizacionais. }\end{array}$ \\
\hline $\begin{array}{l}\text { Gestão de Marcas e Patente } \\
\text { (KOTLER, 2000) }\end{array}$ & $\begin{array}{c}\text { Identifica as criações próprias das organizações, por meio de nome, } \\
\text { símbolos, desenhos, etc. Garante vantagem competitiva e } \\
\text { diferenciação. }\end{array}$ \\
\hline $\begin{array}{l}\text { Gestão de Relacionamento com } \\
\text { Clientes } \\
\text { (SWIFT, 2001; COELHO et al., } \\
\text { 2014) }\end{array}$ & $\begin{array}{c}\text { Comunicações significativas para alavancar as compras, lucratividade, } \\
\text { retenção e lealdade de clientes. Forma de monitorar o ambiente } \\
\text { externo, que visa identificar oportunidades de mercado. }\end{array}$ \\
\hline $\begin{array}{l}\text { Inteligência Competitiva } \\
\text { (SWIFT, 2001; GOMES e BRAGA, } \\
\text { 2004) }\end{array}$ & $\begin{array}{c}\text { Conhecimento prévio das necessidades dos clientes e dos planos dos } \\
\text { concorrentes. Ou seja, antecipa ameaças e identifica oportunidades } \\
\text { para auxiliar na tomada de decisão. }\end{array}$ \\
\hline
\end{tabular}




\begin{tabular}{|c|c|}
\hline $\begin{array}{l}\text { Universidades Corporativas } \\
\qquad \text { (ALPERSTEDT, 2001) }\end{array}$ & $\begin{array}{c}\text { Oferecem cursos valendo créditos, possuem corpo docente e podem } \\
\text { não se restringir apenas ao atendimento dos funcionários. }\end{array}$ \\
\hline $\begin{array}{l}\text { Business Intelligence } \\
\text { (PRIMAK, 2008) }\end{array}$ & $\begin{array}{c}\text { Processo que auxilia na tomada de decisões e gera informações, } \\
\text { através da coleta, organização, análise, compartilhamento e } \\
\text { monitoramento de dados. }\end{array}$ \\
\hline $\begin{array}{l}\text { Lições Aprendidas } \\
\text { (PROBST et al., 2002) }\end{array}$ & $\begin{array}{l}\text { Visa aprender com os sucessos e erros das experiências nos projetos } \\
\text { anteriores, são a essência do conhecimento tácito. }\end{array}$ \\
\hline $\begin{array}{l}\text { Mapeamento de Conhecimentos } \\
\text { (DAVENPORT e PRUSAK, 1999) }\end{array}$ & Retrato daquilo que existe dentro da organização e sua localização. \\
\hline $\begin{array}{l}\text { Mapeamento de Processos } \\
\text { (CHIAVENATO, 1995; COELHO et } \\
\text { al., 2014). }\end{array}$ & $\begin{array}{l}\text { Visualização das interligações e interdependências entre os diferentes } \\
\text { processos empresariais. Mapeia cadeias de valor, processos críticos, } \\
\text { redes de operações e o caminho das atividades que geram o serviço } \\
\text { ou produto. }\end{array}$ \\
\hline $\begin{array}{l}\text { Melhores Práticas } \\
\text { (BATISTA et al., 2005) }\end{array}$ & $\begin{array}{l}\text { Procedimentos validados para realização de tarefas ou solução de } \\
\text { problemas, que devem ser identificados, documentados e difundidos. }\end{array}$ \\
\hline $\begin{array}{l}\text { Memória Organizacional } \\
\text { (PROBST et al., 2002) }\end{array}$ & $\begin{array}{c}\text { Sistema de habilidades e conhecimentos que armazena experiências e } \\
\text { percepções para serem consultadas. É o que possibilita os } \\
\text { aprendizados. }\end{array}$ \\
\hline $\begin{array}{c}\text { Mentoring } \\
\text { (OLIVEIRA, 2018) }\end{array}$ & $\begin{array}{l}\text { Orientação profissional e pessoal pelo mentor experiente e com } \\
\text { conhecimento teórico, para desenvolver habilidades específicas. }\end{array}$ \\
\hline $\begin{array}{l}\text { Normalização e Padronização } \\
\text { (SILVA, 1995) }\end{array}$ & $\begin{array}{l}\text { Redução da variedade de produtos. Institui a fácil comunicação entre } \\
\text { clientes e fornecedores, eliminando barreiras técnicas e comerciais. }\end{array}$ \\
\hline $\begin{array}{l}\text { Portais Corporativos } \\
\text { (TERRA, 2005) }\end{array}$ & $\begin{array}{l}\text { São instrumentos fundamentais para compartilhar informação e } \\
\text { conhecimento no interior das organizações. }\end{array}$ \\
\hline Gestão Eletrônica de & $\begin{array}{c}\text { Utilizam-se aplicativos de controle de emissão, edição, arquivamento, } \\
\text { descarte e outras ações relacionadas aos documentos }\end{array}$ \\
\hline
\end{tabular}




\begin{tabular}{|c|c|}
\hline Documentos & organizacionais. \\
\hline $\begin{array}{c}\text { (BATISTA et al., 2005) } \\
\begin{array}{c}\text { Gestão do Capital Humano } \\
\text { (COELHO et al., 2014) }\end{array}\end{array}$ & $\begin{array}{c}\text { Facilita o mapeamento das competências já existentes ou não. Gera } \\
\text { contexto capacitante para criação e transmissão do conhecimento. }\end{array}$ \\
\hline
\end{tabular}

Fonte: Adaptado de Penteado et al. (2008)

Logo, percebe-se que as práticas valorizam o capital intelectual como meio de transformar o conhecimento tácito em explícito. Ademais, auxiliam na tomada de decisões estratégicas e incentivam a participação dos funcionários e stakeholders rumo a evolução.

\section{METODOLOGIA}

Essa pesquisa enquadra-se em uma abordagem qualitativa, apoia-se na pesquisa bibliográfica para o embasamento teórico e inspira-se em um caso para levantamento de evidências. Nesse sentido, McKay e Marshall (2001) destacam que o posicionamento do pesquisador como membro da organização, como neste trabalho, é crucial, de modo que este se envolva na mudança do problema e gere conhecimento com os resultados obtidos. Logo, a escolha da empresa A como objeto de estudo se deu pela percepção da ausência do nivelamento do conhecimento entre colaboradores na Supervisão de Manutenção em Instrumentação, bem como a consciência de que há poucos estudos de GC na mineração. Além disso, há o fácil acesso à empresa devido a sua localização próxima e ao contato com gestores.

Portanto, para dar consistência à pesquisa, ocorreu a coleta de dados, que pode subdividir-se em dados primários e secundários (MATTAR, 1997). Esse levantamento foi estruturado em quatro fases: definição dos instrumentos de pesquisa; obtenção dos dados primários; obtenção dos dados secundários; tratamento e análise das informações. Sendo assim, para obter os dados primários, foram realizadas observações e entrevistas semiestruturadas com os gestores da área. Para isso, roteiros apoiados pela revisão bibliográfica foram previamente elaborados, de forma a conter tópicos relevantes, mas permitindo a extrapolação para outras discussões pertinentes. Logo, tal revisão bibliográfica, em conjunto com a análise documental, refere-se a captura dos dados secundários. Desse modo, em função da lacuna existente na literatura, foram encontrados 21 documentos, mas apenas 17 foram relevantes para o estudo, devido a mudança de foco dos demais.

A coleta de dados primários foi realizada por meio de entrevistas com dois gestores da área para entender os desafios do setor, os problemas relacionados ao conhecimento e para realizar a validação da estrutura do framework. Para tanto, os gestores responderam às questões referentes ao diagnóstico; identificaram um processo considerado crítico; detalharam as atividades e os cargos envolvidos; apresentaram os conhecimentos associados e discutiram sobre a viabilidade em utilizar algumas práticas de GC. Por fim, foi realizado um tratamento qualitativo de ambos dados, por meio da análise de conteúdo (BARDIN, 2000), passando pelo tratamento, organização, classificação, análise e descrição.

\section{RESULTADOS E DISCUSSÃO}

4.1 Mapeamento na literatura sobre Nivelamento de Conhecimentos e Gestão do Conhecimento na Mineração

Perspectivas em Gestão \& Conhecimento, João Pessoa, v. 11, número especial, p. 97-114, mar. 2021. 
Com o intuito de identificar estudos que abordassem as temáticas nivelamento de conhecimentos e a GC no setor da mineração, foi realizada uma vasta pesquisa bibliográfica.

Sobre a GC na mineração, Ceribeli e Aguiar (2015), diante do desafio de aumentar a eficiência e do investimento em tecnologia da automação e informação, buscaram avaliar como os sistemas de automação podem auxiliar na GC no setor mineração. Para isso, realizaram um estudo de caso em uma mineradora nacional. Já Delgado et al. (2014) aplicaram a Modelagem Orientada à Representação do Pensamento Humano na área do controle de produção da mineradora, com o intuito de compreender o conhecimento adquirido a partir de experiências dos trabalhadores, por meio da identificação de padrões de comportamento constatados por meio de entrevistas. Entretanto, identificaram como fator impeditivo do nivelamento de conhecimentos a enorme quantidade de informações trocadas diariamente entre os funcionários.

Boikanyo et al. (2016), identificaram que a rotatividade e o envelhecimento dos trabalhadores contribuem para a perda de conhecimento tácito no setor da mineração. Ainda, a maior parte dos entrevistados indicaram que não há uma cultura ou infraestrutura técnica propícias para o compartilhamento do conhecimento, embora suas organizações valorizem o conhecimento como estratégia competitiva. Constataram, também, que a GC está correlacionada com os desempenhos de negócio, além da maior concordância dos entrevistados sobre a inexistência da transferência das melhores práticas entre os funcionários. Nesse sentido, Pereira e Barbosa (2018) perceberam que os funcionários da área de engenharia de manutenção industrial de uma mineradora têm acesso a sistemas como os de GC, SISPAV, GED, SAP e portal boas práticas, para solucionar dúvidas e compartilhar o conhecimento. Destacam, ainda, a colaboração na tomada de decisões.

Ainda, Sonntag e Carvalho (2018) partiram da falta da internalização das lições aprendidas na área de projetos da empresa Vale, o que impede que os aprendizados sejam replicados e que não se repitam os mesmos erros. A empresa já contava com uma base de lições para reter, compartilhar os relatos e classificá-los como boas práticas. Todavia, foi perceptível a dificuldade em compartilhar os conhecimentos tácitos individuais, diminuindo a quantidade de lições inseridas na base. Identificou-se que tudo isso ocorreu por conta dos relatos dos funcionários, que não possuíam detalhes suficientes ou não foram bem escritos.

Nesta perspectiva, Brazão e Pech (2018) apresentaram um modelo com base na gestão interativa de lições aprendidas entre projetos similares, que mescla GC e de riscos, para diminuir o tempo de resposta aos riscos. Os autores reconhecem o desafio de implementar mecanismos integrados a GC, dada a dificuldade das pessoas em compartilhar e divulgar informações, do uso errôneo do conhecimento disponível e da má interpretação atrelada à dificuldade em retirar e reutilizar as informações da base de conhecimento.

Quanto ao nivelamento de conhecimentos, foi possível encontrar estudos como o de Fonseca et al. (2013), que dada a dificuldade em se estabelecer o nivelamento do conhecimento nas organizações, propuseram a ferramenta MONK (Módulo de Nivelamento do Conhecimento), no qual há o apoio do processo de disseminação do conhecimento por meio das relações interpessoais em uma organização de software.

Em seu trabalho, Rodrigues Filho e Pasqualin (2011) a fim de nivelar o conhecimento e disponibilizá-lo para consulta didática, sugerem a classificação de informações em uma base de dados com 5 fases (projeto, módulo, perguntas, respostas e justificativas). A alimentação da base seria feita por um especialista da área em questão, com base em perguntas e respostas previamente estruturadas, que possibilitam a transformação da informação em conhecimento.

Por outro lado, Braga et al. (2014) apontam auxiliadores no nivelamento do conhecimento organizacional: treinamentos e reuniões mensais com todos os colaboradores participando na busca de soluções de problemas, onde tudo fica registrado em abertura de planos de ação, atas e relatórios. Outro fator é a distribuição física, que dispõe todos 
trabalhadores juntos no mesmo local, facilitando a interação, colaboração e o aumento da confiança entre a equipe. Todavia, não há documentação dos conhecimentos trocados, o que prejudica os que não estão presentes. Além de estimular a realização de novas tarefas, a empresa incentiva seus funcionários a buscarem cursos, palestras e eventos para se aperfeiçoarem, de forma que logo após repassem seu aprendizado para os que não participaram, por meio de e-mails ou conversas informais. Para os colaboradores, um dos empecilhos do nivelamento são os ruídos na comunicação. Os autores sugeriram a inclusão de metas de transferência do conhecimento no planejamento estratégico, bem como os líderes realizarem a sistematização dos conhecimentos trazidos de fora da empresa.

Nesta linha, variados trabalhos identificaram ou sugeriram práticas de GC com a finalidade de auxiliar no processo de nivelamento de conhecimentos: mentoria, nos quais funcionários mais experientes orientam os novatos e há uma política de retenção do conhecimento (MAKHUBELA; NGOEPE, 2018); marketing interno como ferramenta de contribuição no aumento da cultura do compartilhamento de conhecimentos (MORESI; MENDES, 2010); bibliotecas, salas de conversação, banco de dados, documentos e relatórios em armários de arquivo (BOIKANYO et al., 2016); ferramentas de TI orientadas à GC (MISHRA et al., 2018); ferramenta workflow (SCHMITZ et al., 2013); impressões de jornal mural e reuniões específicas, e-mails e treinamentos (PEREIRA; BARBOSA, 2018); sistema de gerenciamento dos serviços de automação, treinamentos periódicos dos funcionários, auditoria de conhecimento com a identificação de fatores críticos do conhecimento, portal para solucionar problemas com troca de experiências em chats (CERIBELI; AGUIAR, 2015).

$E$, ainda, foi perceptível que, apesar de ser comum a cobrança pela liderança por procedimentos de nivelação do conhecimento nas áreas (PEREIRA; BARBOSA, 2018), há discrepância entre os departamentos de uma mesma empresa quanto ao apoio dos gerentes, recompensas por participação, conhecimento das políticas de retenção existentes e no incentivo da cultura do aprendizado (MAKHUBELA; NGOEPE, 2014).

\subsection{Proposta do Framework Teórico-Analítico}

O setor de Manutenção em Instrumentação e Automação (SMIA) da empresa de mineração denominada, neste trabalho, como empresa $A$, tem como premissa a responsabilidade de garantir a confiabilidade, manutenibilidade e disponibilidade dos ativos de instrumentação. $O$ setor é composto por 15 colaboradores e é dividido em 06 diferentes níveis técnicos, a saber: eletricista 1, eletricista 2, eletricista especializado, técnico 1 , técnico 2 e técnico especializado, tendo cada um uma descrição de cargo e responsabilidade distinta.

Diante da demanda encontrada pelos gestores de urgência na busca de solução em nivelar os conhecimentos dos colaboradores de um mesmo cargo, os elementos das dimensões "Alinhamento com o Negócio" e "Iniciativas de Gestão do Conhecimento" do Modelo de Referência da SBGC (SAITO; FUKUNAGA, 2020) foram utilizadas de forma simplificadas, mas consideradas como norteadores e estratégias eficientes para alcançar este objetivo. Especificamente, até o momento, o framework considerou os seguintes elementos do MR-SBGC: Estratégia de GC, Conhecimentos Relevantes, Processos de GC e Práticas de GC.

Dessa forma, na primeira etapa desta pesquisa foram identificados os principais desafios do negócio e os problemas de GC. Segundo o gestor entrevistado E1, "vários funcionários do setor possuem pouca experiência e baixo nível de conhecimentos técnicos. Com isso, o setor convive com erros repetidos, retrabalho e muito tempo gasto em manutenção corretiva".

A partir deste diagnóstico, foi proposto a identificação do processo considerado mais crítico no setor. O processo escolhido foi o de "Manutenção em Instrumentação" que possui atividades específicas de acordo com o tipo de instrumento. Assim, considerou-se que este

Perspectivas em Gestão \& Conhecimento, João Pessoa, v. 11, número especial, p. 97-114, mar. 2021. 
processo P1 possui subprocessos, a saber P1.1 - Medição da densidade utilizando o Densímetro; P1.2 - Medição de temperatura utilizando o Transmissor de Temperatura; P1.3 Medição de nível Silo/tanque utilizando o Transmissor de Nível; P1.4 - Medição de pressão utilizando o transmissor de pressão; e P1.5 - Medição da vibração para proteção de equipamentos utilizando o Sensor de Vibração.

Posteriormente, foram mapeadas as atividades e os cargos envolvidos em um subprocesso piloto, o P1.4. Assim, identificou-se que o cargo Técnico em Eletroeletrônica executa as seguintes atividades neste subprocesso: (i) montagem e instalação; (ii) configuração do instrumento; (iii) calibração e ajuste; (iv) diagnósticos de falhas; e (v) substituição/reparo em caso de avaria.

Como o objetivo é nivelar os conhecimentos dos colaboradores, a próxima etapa teve o intuito de definir os conhecimentos necessários para a realização das atividades supracitadas pelos colaboradores do cargo Técnico em Eletroeletrônica. Assim, nesta etapa foi realizado a identificação dos conhecimentos críticos (tácitos e explícitos) associados às atividades descritas na terceira etapa. Com isso, os seguintes conhecimentos foram apresentados: o princípio de funcionamento de cada instrumento; a importância dos instrumentos para cada etapa do processo; folha de dados com as especificações técnicas de cada instrumento; e as particularidades das usinas. Nesta etapa, ainda foram consultados os relatórios e sistemas do setor e constatado que estes conhecimentos existem, alguns explicitados e outros, ainda, concentrados em poucos colaboradores.

Contudo, por fim, na quinta etapa foi estabelecido pelos gestores que o processo de GC a ser priorizado pelo setor é o de transferência de conhecimentos, uma vez que os conhecimentos existem, mas não estão acessíveis a todos os colaboradores que executam as atividades mapeadas na terceira etapa. Desse modo, diante da lista de práticas de GC proposta por Batista et al. (2005), os gestores apontaram práticas que já existem e precisam ser adaptadas, e outras que apresentam viabilidade de implementação para nivelamento dos conhecimentos identificados na 4a $\underline{a}$ etapa, como, por exemplo: Educação Corporativa; Comunidade de Prática; Melhores Práticas/Lições Aprendidas; Memória Organizacional; Base de Conhecimentos; Sistemas de Workflow; Storytelling; Mentoria; Gestão de Conteúdo (wiki); Fóruns; e Banco de Competências Individuais.

Dessa forma, finalmente, elabora-se o modelo teórico-analítico (Figura 1), com base nos dados empíricos do caso analisado e no modelo MR-SBGC.

Tem-se, portanto, que diagnosticar os desafios e problemas de GC, mapear os processos críticos, definir cada atividade do processo e os cargos envolvidos, identificar o processo de GC a ser priorizado e selecionar e implementar práticas de GC; sendo etapas cruciais para que as organizações alcancem seus objetivos relacionados ao conhecimento e obtenham melhores resultados. 
Figura 1 - Framework teórico-analítico

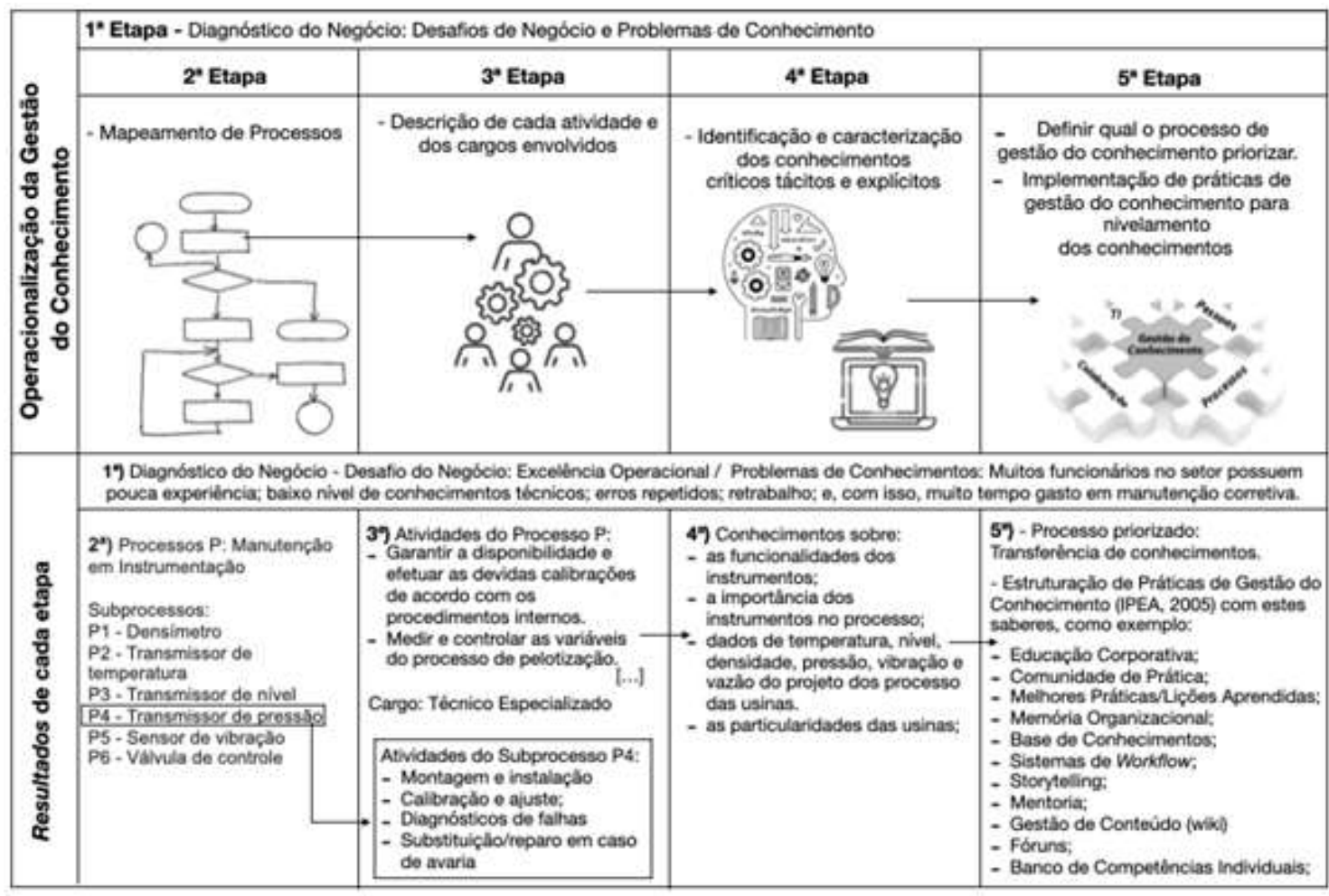

Fonte: Dados da pesquisa

\section{CONSIDERAÇÕES FINAIS}

Com o objetivo de propor um framework teórico-analítico que possibilite às organizações implantarem a gestão do conhecimento para nivelamento dos colaboradores de um mesmo cargo, o presente trabalho realizou uma vasta revisão na literatura de gestão do conhecimento e desta no setor de mineração, a fim de construir pilares conceituais capazes de sustentar as análises empíricas da pesquisa.

Com este efeito, foi possível constatar que os trabalhos não abordam as etapas de implantação da gestão do conhecimento em empresas mineradoras. Diversos trabalhos mostraram a utilização de práticas de GC sem aprofundar na discussão de quais os conhecimentos deveriam, de fato, serem gerenciados. Nesse sentido, com a finalidade de reduzir essa lacuna, este trabalho assumiu como pressuposto que as organizações que carecem nivelar seus conhecimentos devem reconhecer, primeiramente, quais conhecimentos devem ser trabalhados e quais os sujeitos necessitam desses conhecimentos.

Neste sentido, foi elaborado o framework teórico-empírico com as seguintes etapas: diagnosticar os desafios e problemas de gestão do conhecimento; mapear os processos críticos; definir cada atividade do processo e os cargos envolvidos; identificar o processo de gestão do conhecimento a ser priorizado; e selecionar e implementar práticas de GC.

Como principais limitações deste estudo, considera-se que houve a restrição da pesquisa de campo em apenas um único setor desta empresa; o enfoque dado está centrado nos conhecimentos segundo a percepção dos gestores e não dos colaboradores atuantes nas atividades dos processos; as práticas foram apenas selecionadas, sob a ótica dos gestores e, ainda, não foram implementadas para efetiva mensuração de resultados e validação do 
modelo; além do número reduzido de entrevistas realizadas. Contudo, foram entrevistados atores-chave que participam ativamente dos processos decisórios.

Com isso, sugere-se para estudos futuros o acompanhamento e avaliação das práticas de gestão do conhecimento selecionadas, uma vez que espera-se, dentre os inúmeros resultados com a implementação de práticas de gestão do conhecimento na empresa estudada, a sistematização e compartilhamento para que todos colaboradores de um mesmo cargo tenham os conhecimentos críticos e saibam realizar as atividades da mesma forma, dando continuidade ao processo, caso algum colaborador se ausente.

\section{REFERÊNCIAS}

ALPERSTEDT, C. Universidades corporativas: discussão e proposta de uma definição. Revista de Administração Contemporânea, v.5, n. 3, p. 149-165, 2001.

BARDIN, L. Análise de Conteúdo. Lisboa: Edições, v. 70, n. 3, 2009.

BATISTA F. F. et al. Texto para Discussão №1095: Gestão do conhecimento na Administração Pública. Brasília: Instituto de Pesquisa Econômica Aplicada, p. 123, 2005.

BOIKANYO, D. H. et al. Investigating the use of knowledge management as a management tool in the mining industry. Problems and Perspectives in Management, v. 14, n. 1, p. 176-182, 2016.

BRAGA, C. R. de A. et al. Estratégias de sobrevivência e avanço numa organização do terceiro setor: o papel dos capacitadores do conhecimento na Junior Achievement de Minas Gerais. Revista Ibero Americana de Estratégia, v.13, n.1, p. 109-122, 2014.

BRAZÃO, R. R.; PECH, G. Gestão de riscos em projetos similares: um modelo interativo para a captura de lições aprendidas. Iberoamerican Journal of Project Management, v.9, n.1, p. 2447, 2018.

CARVALHO, F. C. A de. Gestão do Conhecimento. São Paulo: Pearson, 2012.

CERIBELI, H. B.; AGUIAR, P. H. M. F. de. Sistemas de automação a serviço da gestão do conhecimento: um estudo de caso no setor de mineração. Revista ADM.MADE, v.19, n. 2, p. 107-128, 2015.

CHAIT, L. P. Se souber, conte a alguém. HSM Management, v. 4, n. 14, p. 122-125, 1999.

COELHO, A. G. de S. et al. Resultado da Produção Organizacional com Base na Utilização da Gestão do Conhecimento. In: SIMPÓSIO DE EXCELÊNCIA EM GESTÃO E TECNOLOGIA, 11., 2014, Resende. Anais [...]. Resende: AEDB, 2014.

DELGADO, S. S. et al. Descrição e aplicação do MORPH para mapear variáveis qualitativas no processo de controle de produção de uma empresa mineradora na região de Itabira/MG. In: SIMPÓSIO ACADÊMICO DE ENGENHARIA DE PRODUÇÃO, 9., 2014, Viçosa. Anais [...]. Viçosa: UFV, 2014.

FONSECA, F. et al. Monk: Uma Ferramenta de Apoio à Socialização do Conhecimento em Equipes de Software. In: SIMPÓSIO BRASILEIRO DE SISTEMAS COLABORATIVOS, 10., 2013, Manaus. Anais [...]. Manaus: SBC, 2013. p. 148-151.

Perspectivas em Gestão \& Conhecimento, João Pessoa, v. 11, número especial, p. 97-114, mar. 2021. 
FREITAS, W. R. S.; JABBOUR, C. J. C. Utilizando estudo de caso(s) como estratégia de pesquisa qualitativa: boas práticas e sugestões. Revista Estudo \& Debate, v.18, n.2, 2011.

GIDDENS, A. As conseqüências da modernidade. 2.ed. São Paulo: UNESP, 1991.

HITT, M. et al. Direct and moderate effects of human capital on strategy and performance in professional service firms: a resource-based perspective. Academy of Management Review, $v$. 44, n. 1, p. 13-28, 2001A.

KUKKO, M. Knowledge sharing barriers in organic growth: a case study from a software company. The Journal of High Technology Management Research, v.24, n. 1, p. 18-29, 2013.

MAKHUBELA, S.; NGOEPE, M. Knowledge retention in a platinum mine in the North West Province of South Africa. South African Journal of Information Management, v. 20, n.1, p. 905-912, 2018.

MATTAR, F. N. Pesquisa de marketing: metodologia e planejamento. 5.ed. São Paulo: Atlas, 1997.

McKAY, J.; MARSHALL, P. The Dual Imperatives of Action Research. Information Technology \& People, v. 14, n. 1, p. 46-59, 2001.

MISHRA, P. C. et al. The Role of Information Technology for Knowledge Management: An Empirical Study of the Indian Coal Mining Industry. Journal of Global Information Technology Management, v. 21, n. 3, p. 208- 225, 2018.

MORESI, E. A. D.; MENDES, S. P. Compartilhamento do conhecimento em portais corporativos. TransInformação, v. 22, n. 1, p. 19-32, 2010.

NONAKA, I.; TAKEUCHI, H. Criação de conhecimento na empresa: como as empresas japonesas geram a dinâmica da inovação. 10.ed. Rio de Janeiro: Campus, 1997.

OLIVEIRA, D. P. R. de. Coaching, Mentoring e Counseling, 3.ed. São Paulo: Atlas, 2018.

PEREIRA, S. L. C. e S.; BARBOSA, E. C. Compartilhamento do conhecimento: uma ferramenta elementar para o bom desenvolvimento de uma empresa. Revista Eletrônica Engenharia de Interesse Social, v. 1, n. 3, p. 1-15, 2018.

PENTEADO, R. F.S. et al. Práticas de Gestão do Conhecimento presentes em um programa de sugestão empresarial. In: SIMPÓSIO ACADÊMICO DE ENGENHARIA DE PRODUÇÃO, 4., 2008, Viçosa. Anais [...]. Viçosa: UFV, 2008.

PRIMAK, F. V. Decisões com BI. Rio de Janeiro: Ciência Moderna, 2008.

PROBST, G.; RAUB, S.; ROMHARDT, K. Gestão do conhecimento: os elementos construtivos do sucesso. Porto Alegre: Bookman, 2002.

QUINN, J. et al. Managing Professional Intellect: Making the Most of the Best. Harvard Business Review, v. 74, n. 2, p. 71-80, 1996. 
RODRIGUES FILHO, J. L.; PASQUALIN, E. C. Gestão do conhecimento sustentável. In: CONGRESSO INTERNACIONAL RESPONSABILIDADE E RECIPROCIDADE, 1., 2012, Recanto Maestro. Atas [...]. Recanto Maestro: Fundação Antonio Meneghetti \& Faculdade Antonio Meneghetti, 2012. p. 239-244.

RUBENSTEIN-MONTANO, B. et al. A systems thinking framework for knowledge management. Decision support systems, v. 31, n. 1, p. 5-16, 2001.

SAITO, A.; FUKUNAGA, F. Modelo de Referência Sociedade Brasileira de Gestão do Conhecimento - SBGC: guia de referência da gestão do conhecimento. São Paulo: SBGC, 2020.

SCHMITZ, Q. et al. Ferramenta Workflow para apoio ao processo de geração de conhecimento: um estudo de casos múltiplos. Produção Em Foco, v. 3, n. 1, p. 96-119, 2013.

SONNTAG, A. A.; CARVALHO, R. B. de. Transferência de Conhecimentos Tácitos para Rotinas Organizacionais: Caso Prático da Área de Projetos da Vale. Revista IPTEC, v. 6, n. 1, p. 64-77, 2018.

SORDI, J. O. de. Administração da Informação: fundamentos e práticas para uma nova gestão do conhecimento. São Paulo: Saraiva, 2008.

SOUZA, A. M.; KURTZ, D. J. Análise de modelos para a gestão do conhecimento organizacional: $\mathrm{O}$ caso serviço social da indústria-SESI/PE. International Journal of Knowledge Engineering and Management (IJKEM), v. 3, n. 6, p. 64-88, 2014.

SPENDER, J. C. Making knowledge the basis of a dynamic theory of the firm. Strategic Management Journal, v. 17, n. S2, p. 45-62, 1996.

STEFANOVITZ, J. P.; NAGANO M. S. Aquisição e criação de conhecimento na indústria de alta tecnologia. Revista Produção Online, v. 6, n. 1, 2006.

SWIFT, R. CRM Customer Relationship Management: O revolucionário marketing de relacionamento com o cliente. Rio de Janeiro: Campus, 2001.

TAKEUCHI, H.; NONAKA, I. Gestão do conhecimento. Porto Alegre: Bookman, 2008.

TEIXEIRA FILHO, J. Gerenciando conhecimento. Rio de Janeiro: SENAC, 2000.

TERRA, J. C. C. Gestão do conhecimento: o grande desafio empresarial. São Paulo: Elsevier, 2005.

WEBER, F. et al. Standardisation in knowledge management - towards a common KM framework in Europe. In: UNICOM SEMINAR, 2002, London. Proceedings [...]. London: 2002.

WIIG, K. Knowledge management foundations. Texas: Schema Press, 1993.

Recebido em/Received: 05/02/2021 | Aprovado em/Approved: 21/02/2021 